\title{
Electronic Properties of Armchair Carbon Nanotubes: Bosonization Approach
}

\author{
Hideo Yoshioka ${ }^{1,2}$ and Arkadi A. Odintsov ${ }^{1,3}$ \\ ${ }^{1}$ Department of Applied Physics, Delft University of Technology, Lorentzweg 1, 2628 CJ Delft, The Netherlands \\ ${ }^{2}$ Department of Physics, Nagoya University, Nagoya 464-8602, Japan \\ ${ }^{3}$ Nuclear Physics Institute, Moscow State University, Moscow 119899 GSP, Russia
}

(Received 11 May 1998)

\begin{abstract}
The phase Hamiltonian of armchair carbon nanotubes at half filling and away from it is derived from the microscopic lattice model by taking the long-range Coulomb interaction into account. We investigate the low-energy properties of the system using the renormalization group method. At half filling, the ground state is a Mott insulator with a spin gap, in which bound states of electrons are formed at different atomic sublattices. [S0031-9007(98)08039-9]
\end{abstract}

PACS numbers: 71.10.Pm, 71.20.Tx, 72.80.Rj

Single wall carbon nanotubes (SWNTs) with diameters of a few atomic distances and lengths of several micrometers can be considered as the ultimate miniaturization of metallic wires [1]. Recent experiments have demonstrated electron transport through individual [2] and multiple [3] SWNTs as well as provide evidence of strong Coulomb interaction in these systems. The one-dimensional nature of the low-energy electronic states in the nanotubes together with the interaction of electrons should result in a variety of correlation effects due to the non-Fermi liquid ground state of the system [4].

Very recent transport spectroscopy data by Tans et al. [5] on spin polarization of an individual SWNT cannot be explained by the constant interaction model and suggests the interpretation in terms of electron correlations. This result, however, was not confirmed by experiments on ropes of SWNTs [6], which fit the constant interaction model remarkably well.

Experimental progress urges the development of a theory of electron correlations in SWNTs. For a model onsite [7] and on-site plus nearest neighbor [8] interactions, metallic armchair SWNTs become Mott insulator at half filling, whereas upon doping they exhibit superconducting fluctuations. The realistic long-range Coulomb interaction was considered in Refs. [9,10]. Kane, Balents, and Fisher [10] discussed the effects of the Coulomb interaction in finite-size armchair nanotubes (ANs) in terms of the Tomonaga-Luttinger low-energy theory. The most important part of the forward scattering was incorporated into the Tomonaga-Luttinger-like Hamiltonian, whereas the other types of scattering were treated as perturbations. Egger and Gogolin (EG) developed an effective low-energy theory of ANs starting from a microscopic model [9], which accounts for all types of scattering processes. They derived a bosonic phase Hamiltonian and discussed possible ground states away from half filling.

In this Letter we derive the phase Hamiltonian of ANs and evaluate its parameters from the microscopic lattice model. The difference between our Hamiltonian and that by EG stems from the distinction in the form of a kinetic term and the use of oversimplified approximation for the $2 k_{F}$ component of scattering amplitudes in Ref. [9]. The renormalization group (RG) method is applied to the Hamiltonian, and the low-energy states are investigated. At half filling the ground state is the Mott insulator with spin gap, in agreement with the conclusion of Hubbardlike models [7,8]. In this state, the electronic bound states are formed between the different sublattices. Away from half filling we predict gaps for both symmetric and antisymmetric spin modes, in contrast to the result of Ref. [9] for the case of equal amplitudes of intrasublattice and intersublattice forward scattering.

We start from the tight-binding single particle Hamiltonian [11] on the honeycomb lattice (inset of Fig. 1),

$$
\mathcal{H}_{k}=\sum_{s, \vec{k}}\left\{\xi(\vec{k}) a_{-, s}^{\dagger}(\vec{k}) a_{+, s}(\vec{k})+\text { H.c. }\right\} .
$$

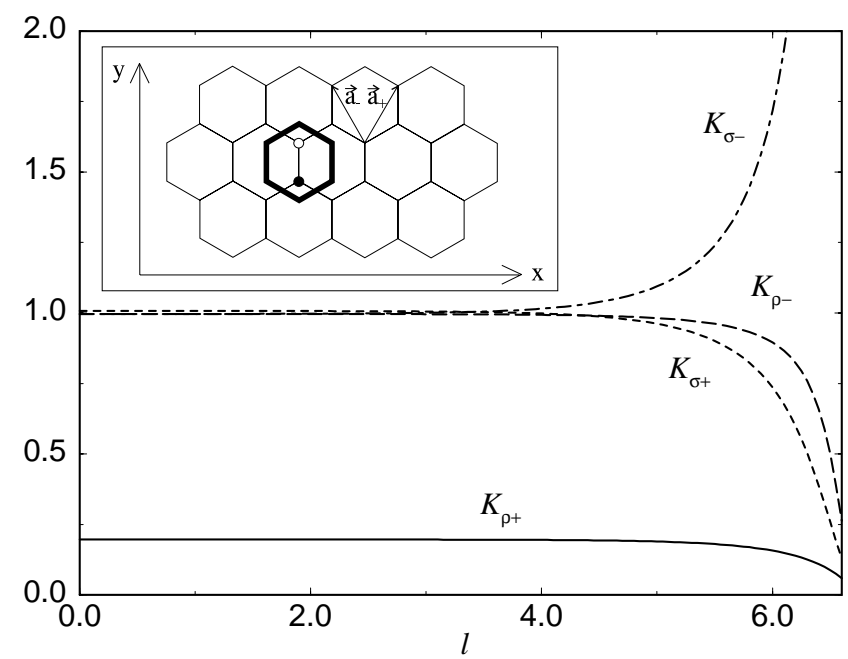

FIG. 1. Solutions of the RG equations for $K_{j \delta}$ in the case of half filling with $N=10, \kappa=1.4, R_{s}=100 \mathrm{~nm}$, and $a_{0}=$ $a / 2$. Inset: The honeycomb lattice of carbon atoms. Here $\vec{a}_{ \pm}$ are the two primitive Bravais lattice vectors, $\left|\vec{a}_{ \pm}\right|=a$. The hexagon shown by the thick line is the unit cell and the black (white) circle denotes the point at $p=+(-)$ sublattice. The $x$ axis points along AN. 
Here $a_{p, s}(\vec{k})$ are the Fermi operators for electrons at the sublattice $p= \pm$ with the spin $s= \pm$ and the wave vector $\vec{k}=\left(k_{x}, k_{y}\right)$. The matrix elements are given by $\xi(\vec{k})=$ $-t\left(e^{-i k_{y} a / \sqrt{3}}+2 e^{i k_{y} a / 2 \sqrt{3}} \cos k_{x} a / 2\right), t$ being the hopping amplitude between neighboring atoms. The eigenvalues of the Hamiltonian vanish at two points of the Brillouin zone, $\vec{k}=\left(\alpha K_{0}, 0\right)$ with $\alpha= \pm$ and $K_{0}=4 \pi / 3 a$, which constitute the Fermi surface of a graphite layer [7,11].

We consider the armchair $(N, N)$ SWNT parallel to the $x$ axis so that the wrapping vector $\vec{w}=N\left(\vec{a}_{+}+\vec{a}_{-}\right)$ points in the $y$ direction (inset of Fig. 1). In this case the Fermi points lie on the allowed quantized transverse wave vector $k_{y}=0$ for any $N$. Expanding Eq. (1) near the Fermi points to the lowest order in $q=k_{x}-\alpha K_{0}$ and introducing slowly varying Fermi fields $\psi_{p \alpha s}(x)=$ $L^{-1 / 2} \sum_{q} e^{i q x} a_{p, s}\left(q+\alpha K_{0}, 0\right)$, we obtain

$$
\mathcal{H}_{k}=-i v_{0} \sum_{p, \alpha, s} \alpha \int d x \psi_{-p \alpha s}^{\dagger} \partial_{x} \psi_{p \alpha s},
$$

$v_{0}=\sqrt{3} \mathrm{ta} / 2 \approx 8 \times 10^{5} \mathrm{~m} / \mathrm{s}$ being the Fermi velocity. It should be noted that the kinetic term (2) differs [12] from the kinetic term used by EG [Eq. (2) of Ref. [9] ].

Following EG, the interaction term reads

$$
\begin{aligned}
\mathcal{H}_{\text {int }}= & \frac{1}{2}\left(\frac{a}{2}\right)^{2} \sum_{l, l^{\prime}} \sum_{p p^{\prime}} \sum_{\alpha_{1} \ldots \alpha_{4}} \sum_{s s^{\prime}} U_{p p^{\prime}}\left(x_{l}-x_{l^{\prime}}\right) \\
& \times e^{i K_{0}\left[\left(\alpha_{4}-\alpha_{1}\right) x_{l}+\left(\alpha_{3}-\alpha_{2}\right) x_{l^{\prime}}\right]} \\
& \times \psi_{p \alpha_{1} s}^{\dagger}\left(x_{l}\right) \psi_{p^{\prime} \alpha_{2} s^{\prime}}^{\dagger}\left(x_{l^{\prime}}\right) \psi_{p^{\prime} \alpha_{3} s^{\prime}}\left(x_{l^{\prime}}\right) \psi_{p \alpha_{4} s}\left(x_{l}\right),
\end{aligned}
$$

with $x_{l}=l a / 2$. The effective $1 \mathrm{D}$ interaction between the sublattices $p$ and $p^{\prime}, U_{p p^{\prime}}\left(x_{l}\right)$, is the average of the Coulomb potential $U(x, y)=$ $e^{2} /\left\{\kappa \sqrt{a_{0}^{2}+x^{2}+4 R^{2} \sin ^{2}(y / 2 R)}\right\}$ over the nodes of a sublattice along the $y$ direction,

$$
U_{p p^{\prime}}\left(x_{l}\right)=\frac{1}{N} \sum_{n} U\left(x_{l}, a \sqrt{3}\left(n+\Delta_{p p^{\prime}}\right)\right),
$$

with $\Delta_{p p^{\prime}}=\bmod (l, 2) / 2+\delta_{p p^{\prime}} / 3$. Here $\kappa$ is an effective dielectric constant of the system (the estimate [9] for the parameters of the experiment [2] gives $\kappa=1.4$ ) and $a_{0} \simeq a$ characterizes the radius of $p_{z}$ orbital.

Equation (3) can be separated into the "forward scattering" $\mathcal{H}_{0}+\mathcal{H}_{f}\left(\alpha_{1}=\alpha_{4}, \alpha_{2}=\alpha_{3}\right)$ and "backscattering" $\mathcal{H}_{b}+\mathcal{H}_{b^{\prime}}\left(\alpha_{1}=-\alpha_{2}=\alpha_{3}=-\alpha_{4}\right)$ [13],

$$
\begin{aligned}
& \mathcal{H}_{0}=\frac{V_{+}(0)}{2} \int d x \rho^{2}(x), \\
& \mathcal{H}_{f}=-\frac{\delta V(0)}{2} \sum_{p \alpha \alpha^{\prime} s s^{\prime}} \int d x \psi_{p \alpha s}^{\dagger} \psi_{-p \alpha^{\prime} s^{\prime}}^{\dagger} \psi_{-p \alpha^{\prime} s^{\prime}} \psi_{p \alpha s},
\end{aligned}
$$

$\mathcal{H}_{b}=\frac{V_{+}\left(2 K_{0}\right)}{2} \sum_{p p^{\prime} \alpha s s^{\prime}} \int d x \psi_{p \alpha s}^{\dagger} \psi_{p^{\prime}-\alpha s^{\prime}}^{\dagger} \psi_{p^{\prime} \alpha s^{\prime}} \psi_{p-\alpha s}$,

$\mathcal{H}_{b^{\prime}}=-\frac{\delta V\left(2 K_{0}\right)}{2} \sum_{p \alpha s s^{\prime}} \int d x \psi_{p \alpha s}^{\dagger} \psi_{-p-\alpha s^{\prime}}^{\dagger} \psi_{-p \alpha s^{\prime}} \psi_{p-\alpha s}$,

where $\rho(x)=\sum_{p \alpha s} \psi_{p \alpha s}^{\dagger} \psi_{p \alpha s}$ is the total electron density, $\quad \delta V(0)=V_{+}(0)-V_{-}(0), \quad$ and $\delta V\left(2 K_{0}\right)=V_{+}\left(2 K_{0}\right)-V_{-}\left(2 K_{0}\right), \quad$ with $\quad V_{ \pm}(q)=$ $(a / 2) \sum_{l} e^{i q x_{l}} U_{p \pm p}\left(x_{l}\right)$.

The forward scattering $\mathcal{H}_{0}$ has the strongest amplitude, $V_{+}(0)=\left(2 e^{2} / \kappa\right) \ln \left(R_{S} / R\right)$, where $R_{S} \simeq \min (L, D)$ characterizes the large distance cutoff of the Coulomb interaction due to a finite length $L$ of the AN and/or the presence of metallic electrodes at a distance $D$ [10]. From Eq. (4), one sees that the amplitudes $\delta V(0)$ and $V_{+}\left(2 K_{0}\right)$ decay as $1 / R$ for $R \gg a$. It should be noted that the matrix element $N V_{-}\left(2 K_{0}\right)$ vanishes identically in the case of a graphite plane $(R \rightarrow \infty)$ due to the $C_{3}$ symmetry of the lattice. For this reason, $V_{-}\left(2 K_{0}\right)$ is much smaller than $\delta V(0)$ and $V_{+}\left(2 K_{0}\right)$. All the matrix elements decrease with increasing $a_{0}$. Numerical evaluation for $a_{0}=$ $a / 2$ and $R \gg a$ gives $\delta V(0)=0.21, V_{+}\left(2 K_{0}\right)=0.60$, $V_{-}\left(2 K_{0}\right)=9.4 \times 10^{-4}$ in units of $a e^{2} / 2 \pi \kappa R\left[V_{-}\left(2 K_{0}\right)\right.$ is estimated for $N=10]$. This result shows that the approximation, $V_{+}\left(2 K_{0}\right)=V_{-}\left(2 K_{0}\right)$, used in Ref. [9] is questionable.

In order to bosonize $\mathcal{H}_{k}+\mathcal{H}_{\text {int }}$, we diagonalize Eq. (2) by the unitary transformation

$$
\psi_{r \alpha s}=\left(\psi_{+\alpha s}+\alpha r \psi_{-\alpha s}\right) / \sqrt{2},
$$

which maps the basis of atomic sublattices $(p= \pm)$ to the basis of right and left movers $(r= \pm)$.

We bosonize the Fermi fields $\psi_{r \alpha s}$,

$\psi_{r \alpha s}=\frac{\eta_{r, \alpha, s}}{\sqrt{2 \pi a}} \exp \left[i r q_{F} x+\frac{i r}{2}\left\{\theta_{\alpha s}+r \phi_{\alpha s}\right\}\right]$,

and decompose the phase variables $\theta_{\alpha s}, \phi_{\alpha s}$ into symmetric $\delta=+$ and antisymmetric $\delta=-$ modes of the charge $\rho$ and $\operatorname{spin} \sigma$ excitations, $\theta_{\alpha s}=\theta_{\rho+}+s \theta_{\sigma+}+$ $\alpha \theta_{\rho-}+\alpha s \theta_{\sigma_{-}}$and $\phi_{\alpha s}=\phi_{\rho+}+s \phi_{\sigma_{+}}+\alpha \phi_{\rho-}+$ $\alpha s \phi_{\sigma^{-}}$. The bosonic fields satisfy the commutation relation, $\left[\theta_{j \delta}(x), \phi_{j^{\prime} \delta^{\prime}}\left(x^{\prime}\right)\right]=i(\pi / 2) \operatorname{sign}\left(x-x^{\prime}\right) \delta_{j j^{\prime}} \delta_{\delta \delta^{\prime}}$. The Majorana fermions $\eta_{r \alpha s}$ satisfy $\left[\eta_{r \alpha s}, \eta_{r^{\prime} \alpha^{\prime} s^{\prime}}\right]_{+}=$ $2 \delta_{r r^{\prime}} \delta_{\alpha \alpha^{\prime}} \delta_{s s^{\prime}}$. The spin-conserving products $\eta_{r \alpha s} \eta_{r^{\prime} \alpha^{\prime} s}$ in the Hamiltonian $\mathcal{H}$ can be represented as [9] $\quad A_{++}(r, \alpha, s)=\eta_{r \alpha s} \eta_{r \alpha s}=1, \quad A_{+-}(r, \alpha, s)=$ $\eta_{r \alpha s} \eta_{r-\alpha s}=i \alpha \sigma_{x}, \quad A_{-+}(r, \alpha, s)=\eta_{r \alpha s} \eta_{-r \alpha s}=$ $\operatorname{ir} \alpha \sigma_{z}$, and $A_{--}(r, \alpha, s)=\eta_{r \alpha s} \eta_{-r-\alpha s}=-i r \sigma_{y}$ with the standard Pauli matrices $\sigma_{i}(i=x, y, z)$. The quantity $q_{F}=\pi n / 4$ is related to the deviation $n$ of the average electron density from half filling and can be controlled by the gate voltage.

The bosonized Hamiltonian has the form 


$$
\begin{aligned}
& \mathcal{H}=\sum_{j=\rho, \sigma} \sum_{\delta= \pm} \frac{v_{j \delta}}{2 \pi} \int d x\left\{K_{j \delta}^{-1}\left(\partial_{x} \theta_{j \delta}\right)^{2}+K_{j \delta}\left(\partial_{x} \phi_{j \delta}\right)^{2}\right\} \\
&+\frac{1}{2(\pi a)^{2}} \int d x\{[\left.\delta V(0)-2 \bar{V}\left(2 K_{0}\right)\right] \cos \left(4 q_{F} x+2 \theta_{\rho+}\right) \cos 2 \theta_{\sigma+}-\delta V(0) \cos \left(4 q_{F} x+2 \theta_{\rho+}\right) \cos 2 \theta_{\rho-} \\
&+\delta V(0) \cos \left(4 q_{F} x+2 \theta_{\rho+}\right) \cos 2 \theta_{\sigma-}-\left[\delta V(0)-\delta V\left(2 K_{0}\right)\right] \cos 2 \theta_{\rho-} \cos 2 \theta_{\sigma-} \\
&+\delta V(0) \cos 2 \theta_{\sigma+} \cos 2 \theta_{\sigma-}-\delta V(0) \cos 2 \theta_{\sigma+} \cos 2 \theta_{\rho-} \\
&-2 \bar{V}\left(2 K_{0}\right) \cos \left(4 q_{F} x+2 \theta_{\rho+}\right) \cos 2 \phi_{\sigma-}+2 \bar{V}\left(2 K_{0}\right) \cos 2 \theta_{\sigma+} \cos 2 \phi_{\sigma-} \\
&\left.+\delta V\left(2 K_{0}\right) \cos 2 \theta_{\rho-} \cos 2 \phi_{\sigma_{-}}+\delta V\left(2 K_{0}\right) \cos 2 \theta_{\sigma-} \cos 2 \phi_{\sigma-}\right\}
\end{aligned}
$$

$v_{j \delta}=v_{0} \sqrt{A_{j \delta} B_{j \delta}}$ and $K_{j \delta}=\sqrt{B_{j \delta} / A_{j \delta}}$ being the velocities of excitations and exponents for the modes $j, \delta$. The parameters $A_{j \delta}, B_{j \delta}$ are given by

$$
\begin{gathered}
A_{\rho+}=1+\frac{4 \bar{V}(0)}{\pi v_{0}}-\frac{\delta V(0)}{4 \pi v_{0}}-\frac{\bar{V}\left(2 K_{0}\right)}{2 \pi v_{0}}-\frac{\delta V\left(2 K_{0}\right)}{4 \pi v_{0}}, \\
A_{\sigma+}=1-\frac{\delta V(0)}{4 \pi v_{0}}-\frac{\bar{V}\left(2 K_{0}\right)}{2 \pi v_{0}}-\frac{\delta V\left(2 K_{0}\right)}{4 \pi v_{0}}, \\
A_{j-}=1-\frac{\delta V(0)}{4 \pi v_{0}}+\frac{\bar{V}\left(2 K_{0}\right)}{2 \pi v_{0}}+\frac{\delta V\left(2 K_{0}\right)}{4 \pi v_{0}}, \\
B_{j \pm}=1+\frac{\delta V(0)}{4 \pi v_{0}} \pm \frac{\bar{V}\left(2 K_{0}\right)}{2 \pi v_{0}} \mp \frac{\delta V\left(2 K_{0}\right)}{4 \pi v_{0}},
\end{gathered}
$$

with $\quad \bar{V}(q)=\left[V_{+}(q)+V_{-}(q)\right] / 2$. The sublatticeindependent forward scattering $\bar{V}(0)$ strongly renormalizes the exponent for the symmetric charge mode, $K_{\rho+} \approx 0.2$ [10], whereas for the other modes the interaction is weak, $K_{j \delta}=1+O(a / R)$ [9].

The bosonized Hamiltonian (8) is different from that derived by EG [9] for the case away from half filling, where the nonlinear terms (8) containing the misfit parameter $q_{F}$ can be neglected due to the breakdown of the momentum conservation. Despite the equal forward scattering parts of both Hamiltonians, there is difference in the backscattering parts. Namely, the Hamiltonian of Ref. [9] can be obtained from ours by substituting $\bar{V}\left(2 K_{0}\right) \rightarrow 0$ and $\delta V\left(2 K_{0}\right) \rightarrow 2 \bar{V}\left(2 K_{0}\right)$. As was already pointed out, EG used the kinetic term different from Eq. (2). Though both the Hamiltonians [Eq. (2) and Eq. (2) of Ref. [9]] have the same energy spectra, the unitary transformations which map the basis of atomic sublattices $(p= \pm)$ to the basis of right and left movers $(r= \pm)$ are different. As a result, the backscattering terms $\mathcal{H}_{b}$ and $\mathcal{H}_{b}^{\prime}$ expressed in the basis of right and left movers are different from those derived by EG. In addition, the approximation, $V_{+}\left(2 K_{0}\right)=V_{-}\left(2 K_{0}\right)$, has been used in Ref. [9]. These factors lead to the difference in the bosonized form of the Hamiltonian.

The low-energy properties of Eq. (8) can be investigated by the RG method [14]. At half filling, $q_{F}=0$, we obtain

$K_{\rho+}^{\prime}=-K_{\rho+}^{2}\left(y_{1}^{2}+y_{2}^{2}+y_{3}^{2}+y_{7}^{2}\right) / 8$,

$K_{\sigma+}^{\prime}=-K_{\sigma+}^{2}\left(y_{1}^{2}+y_{5}^{2}+y_{6}^{2}+y_{8}^{2}\right) / 8$,

$$
\begin{aligned}
K_{\rho-}^{\prime} & =-K_{\rho-}^{2}\left(y_{2}^{2}+y_{4}^{2}+y_{6}^{2}+y_{9}^{2}\right) / 8, \\
K_{\sigma-}^{\prime} & =-K_{\sigma-}^{2}\left(y_{3}^{2}+y_{4}^{2}+y_{5}^{2}\right) / 8+\left(y_{7}^{2}+y_{8}^{2}+y_{9}^{2}\right) / 8, \\
y_{1}^{\prime} & =\left(2-K_{\rho+}-K_{\sigma+}\right) y_{1}-\left(y_{2} y_{6}+y_{3} y_{5}+y_{7} y_{8}\right) / 4, \\
y_{2}^{\prime} & =\left(2-K_{\rho+}-K_{\rho-}\right) y_{2}-\left(y_{1} y_{6}+y_{3} y_{4}+y_{7} y_{9}\right) / 4, \\
y_{3}^{\prime} & =\left(2-K_{\rho+}-K_{\sigma-}\right) y_{3}-\left(y_{1} y_{5}+y_{2} y_{4}\right) / 4, \quad(10) \\
y_{4}^{\prime} & =\left(2-K_{\rho-}-K_{\sigma-}\right) y_{4}-\left(y_{2} y_{3}+y_{5} y_{6}\right) / 4, \\
y_{5}^{\prime} & =\left(2-K_{\sigma+}-K_{\sigma-}\right) y_{5}-\left(y_{1} y_{3}+y_{4} y_{6}\right) / 4, \\
y_{6}^{\prime} & =\left(2-K_{\sigma+}-K_{\rho-}\right) y_{6}-\left(y_{1} y_{2}+y_{4} y_{5}+y_{8} y_{9}\right) / 4, \\
y_{7}^{\prime} & =\left(2-K_{\rho+}-K_{\sigma-}^{-1}\right) y_{7}-\left(y_{1} y_{8}+y_{2} y_{9}\right) / 4, \\
y_{8}^{\prime} & =\left(2-K_{\sigma+}-K_{\sigma-}^{-1}\right) y_{8}-\left(y_{1} y_{7}+y_{6} y_{9}\right) / 4, \\
y_{9}^{\prime} & =\left(2-K_{\rho-}-K_{\sigma-}^{-1}\right) y_{9}-\left(y_{2} y_{7}+y_{6} y_{8}\right) / 4,
\end{aligned}
$$

where ' denotes $d / d \ell$ with $d \ell=d \ln (\tilde{a} / a) \quad(\tilde{a}$ is the new lattice constant). The initial conditions for Eqs. (10) are $K_{j \delta}(0)=K_{j \delta}, \quad y_{1}=[\delta V(0)-$ $\left.2 \bar{V}\left(2 K_{0}\right)\right] / \pi v_{0}, \quad y_{2}=-y_{3}=-y_{5}=y_{6}=-\delta V(0) /$ $\pi v_{0}, \quad y_{4}=-\left[\delta V(0)-\delta V\left(2 K_{0}\right)\right] / \pi v_{0}, \quad y_{7}=-y_{8}=$ $-2 \bar{V}\left(2 K_{0}\right) / \pi v_{0}$, and $y_{9}=\delta V\left(2 K_{0}\right) / \pi v_{0}$. In deriving the RG equations, the nonlinear term $\cos 2 \theta_{\sigma-} \cos 2 \phi_{\sigma-}$ is omitted because this operator stays exactly marginal in all orders and is thus decoupled from the problem [15]. The RG equations away from half filling can be obtained from Eqs. (10) by putting $y_{1}, y_{2}, y_{3}$, and $y_{7}$ to zero. Hereafter we concentrate on the case $N=10, \kappa=1.4$, $R_{S}=100 \mathrm{~nm}$, and $a_{0}=a / 2$ where the initial values of the parameters correspond to the estimates given below Eqs. (5).

Away from half filling, the quantities $K_{\sigma+}$, $K_{\rho-}$, and $K_{\sigma-}^{-1}$ renormalize to zero and the coefficient of $\cos 2 \theta_{\sigma+} \cos 2 \theta_{\rho-} \quad\left(\cos 2 \theta_{\sigma+} \cos 2 \phi_{\sigma-}\right.$ and $\left.\cos 2 \theta_{\rho-} \cos 2 \phi_{\sigma^{-}}\right)$tends to $-\infty \quad(\infty)$. As a result, the phases $\theta_{\sigma_{+}}, \theta_{\rho_{-}}$, and $\phi_{\sigma_{-}}$are locked at $\left(\theta_{\sigma^{+}}, \theta_{\rho^{-}}, \phi_{\sigma^{-}}\right)=(0,0, \pi / 2) \quad$ or $\quad(\pi / 2, \pi / 2,0)$ so that the modes $\sigma \pm$ and $\rho-$ are gapped. In this case, the asymptotic behavior of the correlation functions at $x \rightarrow \infty$ is determined by the correlations of the gapless $\rho+$ mode, $\left\langle e^{i n \theta_{\rho^{+}}(x)} e^{-i n \theta_{\rho^{+}}(0)}\right\rangle \sim x^{-n^{2} K_{\rho_{+}} / 2}$ and $\left\langle e^{i m \phi_{\rho^{+}}(x)} e^{-i m \phi_{\rho^{+}}(0)}\right\rangle \sim x^{-m^{2} / 2 K_{\rho_{+}}} \quad(n=1$ and 2 correspond to $2 q_{F}$ and $4 q_{F}$ density waves and $m=1$ for a superconducting state). Since $K_{\rho+} \approx 0.2$, the $2 q_{F}$ 
density wave correlations seem to be dominant. However, we found that the correlation functions of any $2 q_{F}$ density wave decay exponentially at large distances due to the gapped modes. We therefore are looking for the fourparticle correlations. The $4 q_{F}$ density waves dominate over the superconductivity for $K_{\rho+}<1 / 2$ [16,17]. Such density wave states are given by the product of the charge $n_{ \pm}(x)$ or spin $S_{ \pm}(x)$ densities at different sublattices,

$$
\begin{aligned}
n_{+}(x) n_{-}(x) \sim & -\frac{1}{2(\pi a)^{2}} \cos \left(4 q_{F} x+2 \theta_{\rho+}\right) \\
& \times\left(2 \cos 2 \theta_{\sigma_{+}}+\cos 2 \phi_{\sigma_{-}}-\cos 2 \theta_{\rho_{-}}\right),
\end{aligned}
$$$$
S_{+}(x) S_{-}(x) \sim-\frac{1}{8(\pi a)^{2}} \cos \left(4 q_{F} x+2 \theta_{\rho+}\right)
$$$$
\times\left(2 \cos 2 \theta_{\sigma+}-\cos 2 \phi_{\sigma_{-}}+\cos 2 \theta_{\rho-}\right),
$$

where we neglected the unlocked phases $\phi_{\sigma_{+}}, \phi_{\rho_{-}}, \theta_{\sigma_{-}}$ whose contribution decays exponentially at large distances. Substituting the values of the locked phases we observe that $n_{+}(x) n_{-}(x)$ vanishes, and the dominant state is the $4 q_{F}$ spin density wave with correlation function $\left\langle S_{+}(x) S_{-}(x) S_{+}(0) S_{-}(0)\right\rangle \sim \cos 4 q_{F} x / x^{2 K_{\rho+}}$.

The modes $\sigma \pm$ and $\rho$ - remain gapped also in the limit $\delta V(0)=0$. In this case EG have obtained that the symmetric modes, $\rho+$ and $\sigma+$, are gapless, whereas the $\rho-$ mode is gapped and the $\sigma-$ mode separates into the gapless and gapped parts. The result by EG follows from the special dual symmetry $\theta_{\sigma^{-}} \leftrightarrow \phi_{\sigma^{-}}$of the Hamiltonian and the absence of nonlinear terms in the $\rho+$ and $\sigma+$ sectors. Both these factors are lacking in Eq. (8). On the other hand, the result by EG for a finite value of $\delta V(0)$ is qualitatively the same as ours.

At half filling the solution (Fig. 1) of the RG equations (10) indicates that the phase variables $\theta_{\rho+}, \theta_{\sigma+}$, $\theta_{\rho_{-}}$, and $\phi_{\sigma_{-}}$are locked and all kinds of excitation are gapped. In other words, the ground state of the half filled AN is a Mott insulator with spin gap. The same conclusion has been drawn from the model with short-range interactions $[7,8]$. The locked phases are given by $\left(\theta_{\rho+}, \theta_{\sigma_{+}}, \theta_{\rho_{-}}, \phi_{\sigma_{-}}\right)=(0,0,0,0)$ or $(\pi / 2, \pi / 2, \pi / 2, \pi / 2)$ since the coefficients tend to $-\infty$ for the first, second, and sixth through ninth nonlinear terms in Eq. (8). The averages $\left\langle n_{+}(x) n_{-}(x)\right\rangle$ and $\left\langle S_{+}(x) S_{-}(x)\right\rangle$ are both finite, which indicates the formation of bound states of electrons at different sublattices.

The states derived from the present analysis are characteristic for the long-range Coulomb interaction. In fact, for the on-site plus nearest neighbor interaction the dominant states correspond to the density waves at half filling and to the superconducting state or the density waves away from it [8].

The gap $\Delta_{\rho+}$ in the $\rho+$ mode is estimated by the selfconsistent harmonic approximation as [18]

$$
\Delta_{\rho+} /\left(2 v_{\rho+} a^{-1}\right)=\left[\frac{K_{\rho+}}{\pi v_{0}} \sqrt{\frac{\delta V(0)^{2}}{2}+\bar{V}\left(2 K_{0}\right)^{2}-\delta V(0) \bar{V}\left(2 K_{0}\right)}\right]^{1 /\left(1-K_{\rho+}\right)} .
$$

Using the value of the matrix elements calculated numerically, the charge gap for $N=10$ is estimated as $\sim 100 \mathrm{~K}$ for $a_{0}=a / 2$ and $\sim 10 K$ for $a_{0}=a$ [in the case of $a_{0}=$ $a, \delta V(0)=5.6 \times 10^{-3}$ and $\bar{V}\left(2 K_{0}\right)=6.9 \times 10^{-2}$ in units of $\left.a e^{2} / 2 \pi \kappa R\right]$. At high temperatures, $T \gg \Delta_{\rho+}$, the perturbation theory with respect to the nonlinear terms of the Hamiltonian (8) gives the following result for the resistivity $\rho$ at half filling, $\rho \sim T^{2 K_{\rho^{+}}-1} / N^{2}$. On the other hand, $\rho \propto \exp \left(\Delta_{\rho+} / T\right)$ at $T \ll \Delta_{\rho+}$ [10]. The temperature dependence of the resistivity at half filling is a characteristic signature of the Mott transition. We conjecture that this signature can be best detected in multiprobe transport measurements [19].

The authors thank G. E. W. Bauer, R. Egger, and Yu. V. Nazarov for stimulating discussions and L. Balents for a critical reading of the manuscript. The financial support of the Dutch Foundation for Fundamental Research on Matter (FOM) is gratefully acknowledged. This work is also a part of INTAS-RFBR 95-1305.

[1] A. Thess et al., Science, 273, 483 (1996).

[2] S. J. Tans et al., Nature (London) 386, 474 (1997).

[3] M. Bockrath et al., Science 275, 1922 (1997).
[4] For a recent review, see J. Voit, Rep. Prog. Phys. 58, 977 (1995).

[5] S. J. Tans et al., Nature (London) 394, 761 (1998).

[6] D. H. Cobden et al., Phys. Rev. Lett. 81, 681 (1998).

[7] L. Balents and M. P. A. Fisher, Phys. Rev. B 55, R11973 (1997).

[8] Yu. A. Krotov, D.-H. Lee, and Steven G. Louie, Phys. Rev. Lett. 78, 4245 (1997).

[9] R. Egger and A. O. Gogolin, Phys. Rev. Lett. 79, 5082 (1997).

[10] C. Kane, L. Balents, and M. P. A. Fisher, Phys. Rev. Lett. 79, 5086 (1997).

[11] P. R. Wallace, Phys. Rev. 71, 622 (1947).

[12] In fact, the kinetic term of Ref. [9] corresponds to the $y$ component of Eq. (1) which would describe a conducting zigzag nanotube parallel to the $y$ axis.

[13] Note that two-particle "Umklapp" scattering, $(\alpha, \alpha) \leftrightarrow$ $(-\alpha,-\alpha)$, proposed in Ref. [9], is forbidden due to violation of the quasimomentum conservation.

[14] T. Giamarchi and H. J. Schulz, J. Phys. (Paris) 49, 819 (1988); Phys. Rev. B 37, 325 (1988); 39, 4620 (1989).

[15] R. Egger and A. O. Gogolin, Eur. Phys. J. B 3, 281 (1998).

[16] N. Nagaosa, Solid State Commun. 94, 495 (1995).

[17] H. J. Schulz, Phys. Rev. B 53, R2959 (1996).

[18] The same exponent has been obtained in Ref. [10].

[19] A. Bezryadin et al., Phys. Rev. Lett. 80, 4036 (1998). 